\title{
Role of High-Resolution CT and MRI in Predicting the Degree of Difficulty in Patients undergoing Cochlear Implant Surgery: An Institutional Experience
}

\author{
Preeti Tiwari ${ }^{1}$ Sheo Kumar ${ }^{1}$ Prabhaker Mishra² \\ Archana Gupta ${ }^{1}$ Amit Keshri ${ }^{3}$ \\ ${ }^{1}$ Department of Radio Diagnosis, Sanjay Gandhi Postgraduate \\ Institute of Medical Sciences, Lucknow, Uttar Pradesh, India \\ ${ }^{2}$ Biostatistics and Health Informatics, Sanjay Gandhi Postgraduate \\ Institute of Medical Sciences, Lucknow, Uttar Pradesh, India \\ ${ }^{3}$ Division of Neuro-otology, Department of Neurosurgery, Sanjay \\ Gandhi Postgraduate Institute of Medical Sciences, Lucknow, \\ Uttar Pradesh, India
}

Ann Otol Neurotol:2020;3:1-9
Rajat Jain ${ }^{3} \quad$ Namita Mohindra ${ }^{1}$

\begin{abstract}
Address for correspondence Amit Keshri, MS (ENT), Neuro-otology Unit, Department of Neurosurgery, Sanjay Gandhi Postgraduate Institute of Medical Sciences, Neurosurgery Office, C Block, 1st Floor, Lucknow 226014, Uttar Pradesh, India (e-mail: amitkeshri2000@yahoo.com).
\end{abstract}

\begin{abstract}
Introduction Preoperative imaging is a standard practice for cochlear implant candidacy. We are discussing association between surgical feasibility and various anatomical details of middle and inner ear by both high-resolution computed tomography (HRCT) and $3 \mathrm{~T}$ magnetic resonance imaging (MRI) in patients with bilateral sensorineural hearing loss (SNHL) and are used as a guide during surgery as well as foresee complications in cochlear implantation.

Materials and Methods A total of 56 patients of aged 1 to 5 years (prelingual) and $>1$ years (postlingual or perilingual) having bilateral SNHL were included in the study. HRCT temporal bone and MRI head was done in all candidates. Based on the imaging findings of CT and MRI, patients were divided into two categories (normal and abnormal). Demographic and clinical values were compared between two groups. Binary logistic regression analysis was used to identify the predictors of the outcome variable (surgical difficulty).

Results Out of 56 patients, 55 underwent cochlear implantation by Veria technique, one patient having Michel's deformity was denied surgery and was advised brain stem implant. Note that $69.1 \%$ patients had no radiological abnormality, while $30.9 \%$ patients showed abnormality including acquired disease and malformation. Out of the total study patients, $18 \%(n=10)$ showed various type of congenital inner ear malformation. Large size of the external auditory canal (EAC), high basal turn angle (BTA), and increased distance between tympanic segment of facial canal to EAC (midpoint between level of I-S joint corresponding to EAC and exit of chordae tympanic at level

Keywords

- cochlear implant

- imaging

- HRCT

- MRI

- temporal bone

- Veria technique

- basal turn angle

of EAC), all these factors showed inverse relationship with difficulty in surgery, which were found to be statistically significant.

Conclusion The imaging in cochlear implant patients is an essential tool for preoperative assessment of candidacy, surgical planning, and avoid intra- and postoperative complications. BTA and distance between tympanic segment of facial nerve and EAC (midpoint between level of I-S joint corresponding to EAC and exit of chordae tympanic at level of EAC) are important predictors for evaluating intra- and postoperative complications.
\end{abstract}

DOI https://doi.org/ 10.1055/s-0040-1715552 ISSN 2581-9607.
Copyright @2020 Indian Society of Otology
License terms

() (1) $\Theta \circledast$ 


\section{Introduction}

Inner ear hearing loss is the second most prevalent chronic disease worldwide after arthritis. Incidence of sensorineural hearing loss (SNHL) in adult population of western nations is almost $15 \% .{ }^{1}$ The prevalence of deafness in Southeast Asia ranges from 4.6 to $8.8 \%$. In India, 63 million people (6.3\%) suffer from significant auditory loss. Nationwide disability surveys have estimated that hearing loss is the second most common cause of disability. Cochlear implant is surgically implanted electronic device that provides a sense of sound to a person who is profoundly deaf or severely hard of hearing in both ears. Cochlear implantation has become an accepted treatment for severe to profound deafness in patients who derive only minimal benefit from conventional amplification. In India, although the number of children having bilateral SNHL is high, still cochlear implantation is not commonly done due to the high costs for the implant, as well as subsequent speech therapy. ${ }^{2}$ It is important to be familiar with the various available imaging options and with findings that could significantly impact or even preclude implantation. High-resolution computed tomography (HRCT) and magnetic resonance imaging (MRI) are regularly used for cochlear implant preoperative evaluation for the evaluation of inner ear malformations, surgical planning, and to prevent intraoperative complications. In this study, we have discussed the various anatomical details of middle and inner ear by both CT and MRI in patients having bilateral SNHL which have an important role and are used as a guide during the surgery as well as prevent complications in cochlear implantation by Veria technique.

\section{Objectives}

- To determine the various radiological measurements through high-resolution imaging (CT and MR) for cochlear implantation candidacy.

- Association between imaging findings and the level of difficulty encountered during surgery by Veria technique.

\section{Materials and Methods}

Prospective study was conducted in a tertiary care center in North India from July 2015 to December 2017. A total of 55 patients were included in the study. After approval from institutional ethic committee, informed consent was taken from the patients. HRCT temporal bone and MRI head was done in cochlear implant candidates.

\section{Inclusion Criteria}

A prime candidate for inclusion is described as:

- Age 1 to 5 years for prelingual and $>1$ year for postlingual and perilingual.

- Having severe to profound SNHL in both ears.

- Family willing to work toward speech and language skills with therapy.

\section{Exclusion Criteria}

The exclusion criteria were: eMedically unfit for surgery.

- Age $<1$ year.

- Patient (parents in case of children) not consenting for the study.

\section{Imaging Protocol}

HRCT temporal bone and MRI images were evaluated in axial planes reconstructed parallel to the long axis of the lateral semicircular canals (SCCs). Coronal and sagittal sections were viewed perpendicular to the plane of the axial images. Imaging of pediatric population was performed under sedation or short-acting general anesthesia and both CT scan and MRI was done under single sedation.

\section{HRCT Temporal Bone Protocol}

64-detector row CT scanner (Brilliance 190 P 64; Phillips Medical Systems, Netherlands) was used for all HRCT investigations. The patient lay in supine position. No gantry tilt was used to facilitate free reconstructions of the images. All the images was reviewed with a high-resolution bone algorithm for separate documentation of the right and left ears. Axial images were obtained from the apex of the petrous bone to the inferior tip of the mastoid. Coronal reformatted images were obtained from the anterior margin of the petrous apex to the posterior margin of the mastoid. On a multidetector CT scanner, the raw axial image data set was reconstructed with a section thickness of as low as $0.3 \mathrm{~mm}$ with bone windows setting. Low-dose CT scan could have been performed but because of the dense petrous bone and high-resolution requirement, quality of images would have been compromised. No contrast injection was given.

\section{MRI Protocol}

In the same session, all patients underwent MRI on 3T MR scanner (Signa HDXT, GE Healthcare, United States) by using head coil. Patients were positioned in the supine position with head in the head coil and 3-plane localizer was obtained. Axial scanning was performed from vertex to the line of foramen magnum in a plane parallel to the anterior and posterior margin of corpus callosum (AC-PC line). Routine brain sequences were acquired to display any brain pathology and central acoustic pathway anatomy from the cochlear nuclei to the temporal acoustic area. Axial T2-weighted PROPELLAR was done with the following parameters: TR/TE/, 5600/95.5/Ef; NEX, 1; slice thickness, $5 \mathrm{~mm}$; gap, 0.5 ; matrix, $320 \times 320$; field of view (FOV), $24 \mathrm{~cm}$. Axial T1-weighted fluid-attenuated inversion recovery (FLAIR) sequence was done with the following parameters: TR/TE/, 1254/11.6/Ef; NEX, 1; slice thickness, $5 \mathrm{~mm}$; gap 0.5; matrix,512 $\times 256$; FOV, $24 \mathrm{~cm}$. Axial T2 FLAIR sequence was taken with the following parameters: TR/TE/, 8802/85.4/Ef; NEX, 1; slice thickness, $5 \mathrm{~mm}$; gap, 0.5; matrix, $256 \times 160$; FOV, $24 \mathrm{~cm}$. Axial diffusion-weighted 1 sequence was used with the following parameters: TR/TE/, 5600/72.4/FE; NEX, 1; slice thickness, $5 \mathrm{~mm}$; gap, 0.5; matrix, $128 \times 160 ; \mathrm{FOV}, 24 \mathrm{~cm}$. In addition 
to above sequences, following specific MRI protocol was used for evaluation of inner ear anatomy and cochlear nerve. Axial three-dimensional (3D) fast imaging enabling steady-state acquisition (FIESTA) sequence (TR/TE/, 4.8/1.8/ Fr; NEX, 2; slice thickness, $1 \mathrm{~mm}$; gap,-0.5 overlapping; matrix, $320 \times 256$; FOV, $20 \mathrm{~cm}$ ) was performed to cover internal auditory canal (IAC) from hippocampus up to the C-1 vertebral body. Oblique sagittal reformations were done along the plane perpendicular to the course of the seventh and eighth nerves in the IAC and cerebellopontine angle with 3D maximum intensity projection reconstructions. A 3D FIESTA sequence was also acquired in a coronal plane parallel to the line along the right and left IAC to cover the IAC from posterior wall of sphenoid sinus up to the line of fourth ventricle. Postcontrast sequence was not needed. The total scanning time was approximately 20 to 25 minutes for each patient.

Basal turn angulations: To estimate the rotated/tilting/ misaligned cochlea, we have measured an angle called the basal turn angle (BTA). We have drawn two lines:

- First line parallel to the basal turn of cochlea.

- Second line passing through the sagittal mid-plane.

Then the angle was measured between these two lines (-Fig. 1).

\section{Imaging Findings}

We have grouped the patients into two categories (i.e., normal and abnormal) based on the imaging findings on CT and MRI. Patients with acquired disease in ear and congenital

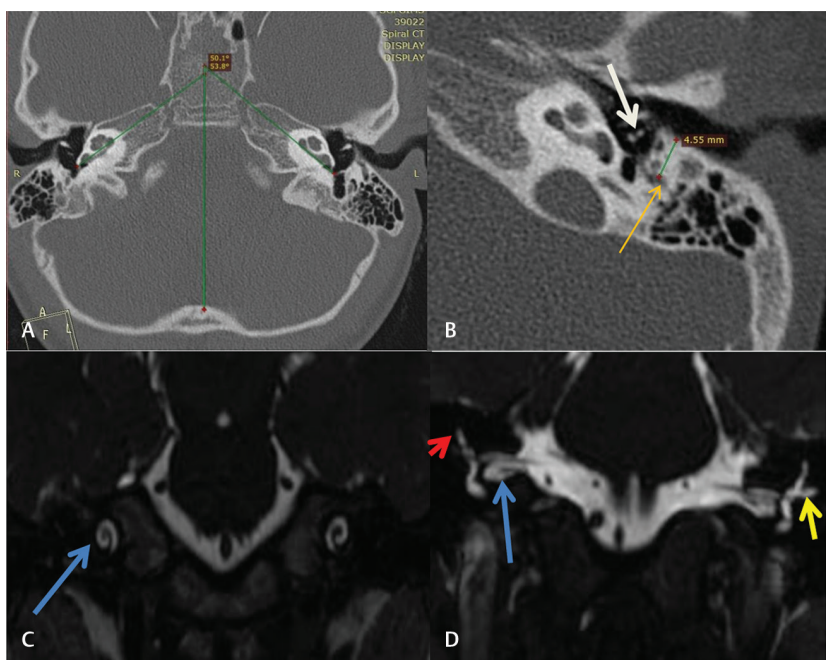

Fig. 1 (A) Computed tomography (CT) scan (axial view of temporal bone): right, basal turn angle (BTA) of 53.8 degrees, and left, BTA of 50.1 degrees. (B) Distance between the tympanic segment of facial nerve and posterior wall of external auditory canal (EAC) at the level of incudostapedial joint $(4.55 \mathrm{~mm})$ (white arrow: I-S joint, orange arrow: tympanic segment of facial nerve). (C) Coronal fast imaging enabling steady-state acquisition (FIESTA) magnetic resonance imaging (MRI) image showed normal bilateral cochlear turns (arrows). (D) Coronal FIESTA MRI image showed normal bilateral internal auditory canal (IAC) (blue arrow) with normal vestibule-cochlear complex, vestibule, and superior (red arrow) and lateral (yellow arrow) semicircular canal. inner ear malformation were considered as abnormal imaging finding.

Surgical difficulty: Surgical difficulty score of all the patients who underwent surgery was assessed by the operating surgeon on 0 to 10 scales, where 10 was most difficult score. Later, the score was divided into three groups where 0 to 4 was considered no difficulty, 5 to 8 mild to moderate difficulty, and $\geq 9$ severe difficulty. For the final analysis, overall patients were divided into two groups, that is, difficult $(\geq 5)$ and not difficult $(\leq 4)$.

\section{Statistical Analysis}

Normality of the continuous data was tested using $Z$ score and variables were considered normal when $Z$ score was within \pm 3.29 . Descriptive statistics of the continuous variable was presented in mean \pm standard deviation/median (interquartile range) while categorical variable in frequency and percentages. To compare the means between two groups, independent samples $t$-test was used, while for nonnormal data, Mann-Whitney $U$ test was used. To test the association/ compare the proportions between two variables, chi-square test/Fisher's exact test was used as appropriate. Univariate and multivariate binary logistic regression analysis was used to find out the predictors of the surgical difficulty. Odds ratio and adjusted odds ratio (AOR) of the surgical difficulty was calculated for the predictors. All the significant variables (predictors) found in multivariate analysis for the surgical difficulty were used in receiver operating characteristics (ROC) curve analysis. Area under curve (AUC) and appropriate cutoff values was selected to identify the surgical difficulty with corresponding sensitivity and specificity. Minimum 95\% confidence interval (CI) or $p$-value $<0.05$ was considered as statistically significant. Statistical Package for Social Sciences, version 23 (SPSS-23, IBM, Chicago, United States) was used for data analysis.

\section{Results}

Fifty-six patients having bilateral SNHL were included in the study. Out of these 56 patients, 55 patients had undergone imaging followed by the cochlear implants by Veria technique (-Fig. 2), while 1 patient who was diagnosed having Michel's deformity was referred for brain stem implant. Patients age ranged between 1 and 48 years with maximum patients in the age group of $\leq 5$ years (prelingual) $(n=31,56.4 \%)$ followed by $>10$ years $(n=14,25.5 \%)$ and 6 to 10 years $(n=10,18.2 \%)$ with a gender distribution of 29 males (52.7\%) and 26 (47.3\%) females. There was significant ( $p=0.016$ ) difference in percentage of male patients between three age groups, that is, $41.5 \%$ ( $\leq 5$ years), $40 \%$ (6-10 years), and $85.7 \%$ (> 10 years). In this study, out of 17 patients, those showing abnormality, 7 patients showed some type of acquired disease which included otomastoiditis $(n=5)$ and otosclerosis $(n=2)$, while 10 patients showed various type of congenital inner ear malformation (-Table 1). Out of 10 cases having congenital inner ear malformation, Mondini's dysplasia, type I incomplete 


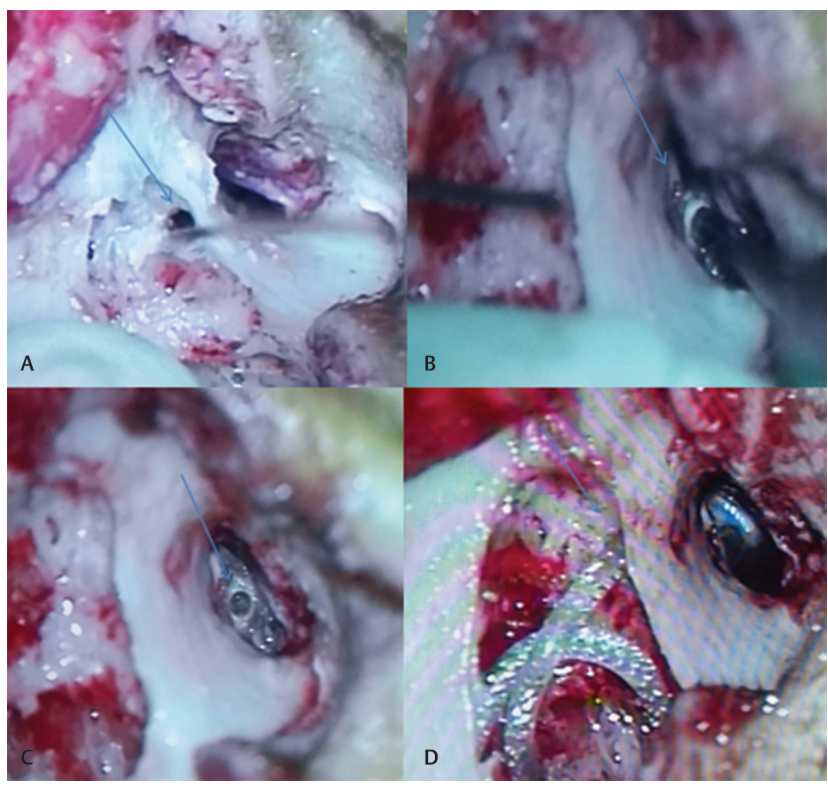

Fig. 2 Veria technique. (A)Tunnel made parallel to external auditory canal (EAC) using Trifon bur. (B) Cochleostomy through EAC after raising tympano-meatal flap. (C) Cochleostomy. (D) Electrodes passed trough tunnel made parallel and inserted into cochlea through cochleostomy.

Table 1 Imaging findings in the study patients

\begin{tabular}{|c|c|c|c|}
\hline \multicolumn{2}{|r|}{ Findings } & \multicolumn{2}{|c|}{ Number of patients $(N=55)$} \\
\hline \multicolumn{2}{|l|}{ Normal } & \multicolumn{2}{|c|}{$38(70.18 \%)$} \\
\hline \multirow[t]{5}{*}{ Abnormal } & Otomastoiditis & 5 & \multirow[t]{5}{*}{$17(29.82 \%)$} \\
\hline & Otosclerosis & 2 & \\
\hline & Poor mastoid & 6 & \\
\hline & High jugular bulb & 4 & \\
\hline & Congenital inner ear & 10 & \\
\hline
\end{tabular}

Note: In abnormal imaging finding, multiple abnormality is included.

partition ( - Fig. 3 ), and bilateral enlarged vestibular aqueduct were found in 3 of 3 cases (each 30\%) while Michel deformity was found in 1 case (10.0\%) (-Table 2, - Fig. 4). The above radiological outcomes was insignificantly correlated with age, sex, BTA, external auditory canal (EAC), and IAC measurements of the candidates $(p>0.05)$.

\section{Distance between the Tympanic Segment of Facial Canal and Post Wall of EAC}

We measured the distance between the tympanic segment of facial canal and posterior wall of EAC (midpoint between level of I-S joint corresponding to EAC and exit of chordae tympani nerve at the level of EAC) in 55 studied cases. Of the 55 cases, 54 (98.2\%) showed that the distance was more than $3 \mathrm{~mm}$ with a mean of $4.55 \pm$ $0.57 \mathrm{~mm}$ (-Fig. 5). Only in one case $(1.8 \%)$ who had congenital inner ear malformation (Michel deformity), the distance was $2.7 \mathrm{~mm}$. It was also found that the mean distance was greater in older ( $>5$ years, $4.58 \pm 0.62 \mathrm{~mm}$ ) than younger age group ( $\leq 5$ years, $4.55 \pm 0.49 \mathrm{~mm}$ ), although the difference was not statistically significant
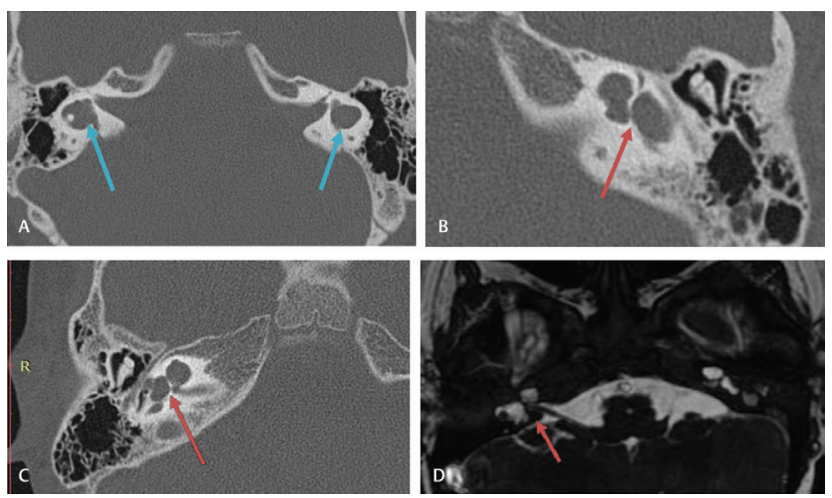

Fig. 3 (A-C) Axial high-resolution computed tomography (HRCT) and (D) magnetic resonance imaging (MRI) axial fast imaging enabling steady-state acquisition (FIESTA) showed cystic bilateral vestibule and cochlea (8 appearances)-finding S/O type I incomplete partition defect.

Table 2 Distribution of congenital malformation in the patients

\begin{tabular}{|l|l|}
\hline Type & No. of cases \\
\hline Michel deformity & $1(10.0 \%)$ \\
\hline Type I incomplete partition & $3(30.0 \%)$ \\
\hline Mondini's dysplasia & $3(30.0 \%)$ \\
\hline $\begin{array}{l}\text { Bilateral enlarged vestibular } \\
\text { aqueduct syndrome }\end{array}$ & $3(30.0 \%)$ \\
\hline Total & $10(100 \%)$ \\
\hline
\end{tabular}
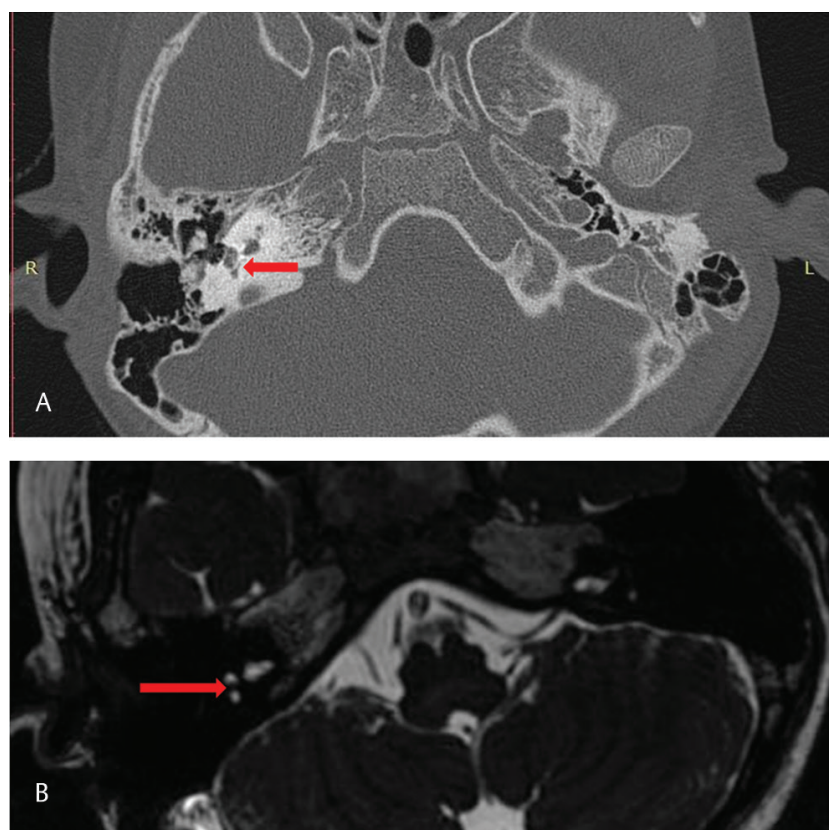

Fig. 4 Michel deformity. (A) High-resolution computed tomography (HRCT) axial absence of normally visualized vestibule and cochlear turns in right petrous temporal bone (arrow). Corresponding (B) magnetic resonance imaging (MRI) axial fast imaging enabling steady-state acquisition (FIESTA) image of same patient showed findings similar to HRCT.

(independent samples $t$-test, $p=0.860$ ). In patient having inner ear malformation, mean was $3.90 \pm 0.44 \mathrm{~mm}$. 
In patients having acquired disease, mean distance was $4.30 \pm 0.47 \mathrm{~mm}$. Similarly, coronal FIESTA MRI image was used to show normal bilateral cochlear turns and coronal FIESTA MRI image for normal bilateral IAC with normal vestibule-cochlear complex, vestibule, and superior and lateral SCC.

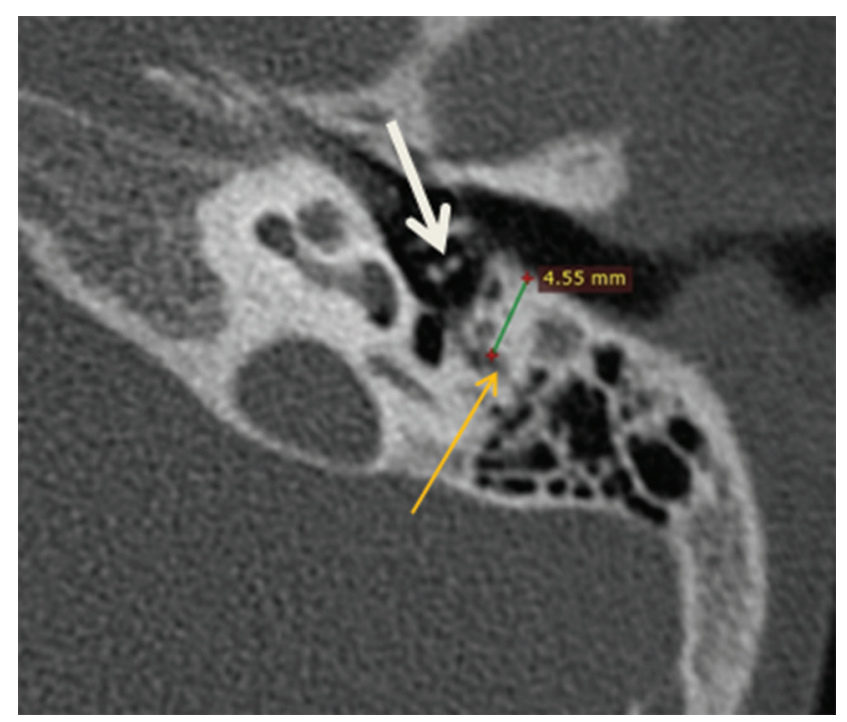

Fig. 5 Distance between tympanic segment of facial nerve and posterior border of external auditory canal (EAC).

\section{Comparisons between Surgical Groups (Difficult) Smooth)}

Out of the 55 patients who had undergone surgery, 13 (23.6\%) patients' surgery was marked as difficult (11 patients associated with mild to moderate difficulty and 2 patients with severe difficulty) (-Fig. 6). Age and sex of the patients were compared between two surgical groups (smooth and difficult), and we found that there was no significant difference in distribution of age $(p=0.359)(-$ Fig. 7$)$ and proportions of male/females $(p=0.926)$ between the two surgical groups ( - Table 3$)$.

The mean score of BTA $(p=0.032)$, EAC $(p=0.004)$, and IAC $(p=0.009)$ and the distance between tympanic segment

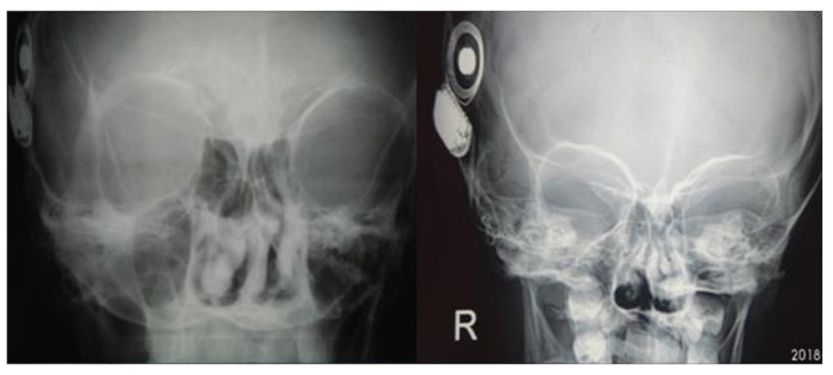

Fig. 6 Difficulty was experienced in terms of insertion and implantation of internal auditory canal (IAC). Postoperative X-ray transorbital view with cochlear implant in situ. Right side image shows cochlear implant electrodes in IAC, left side image shows cochlear implant electrodes in cochlea.

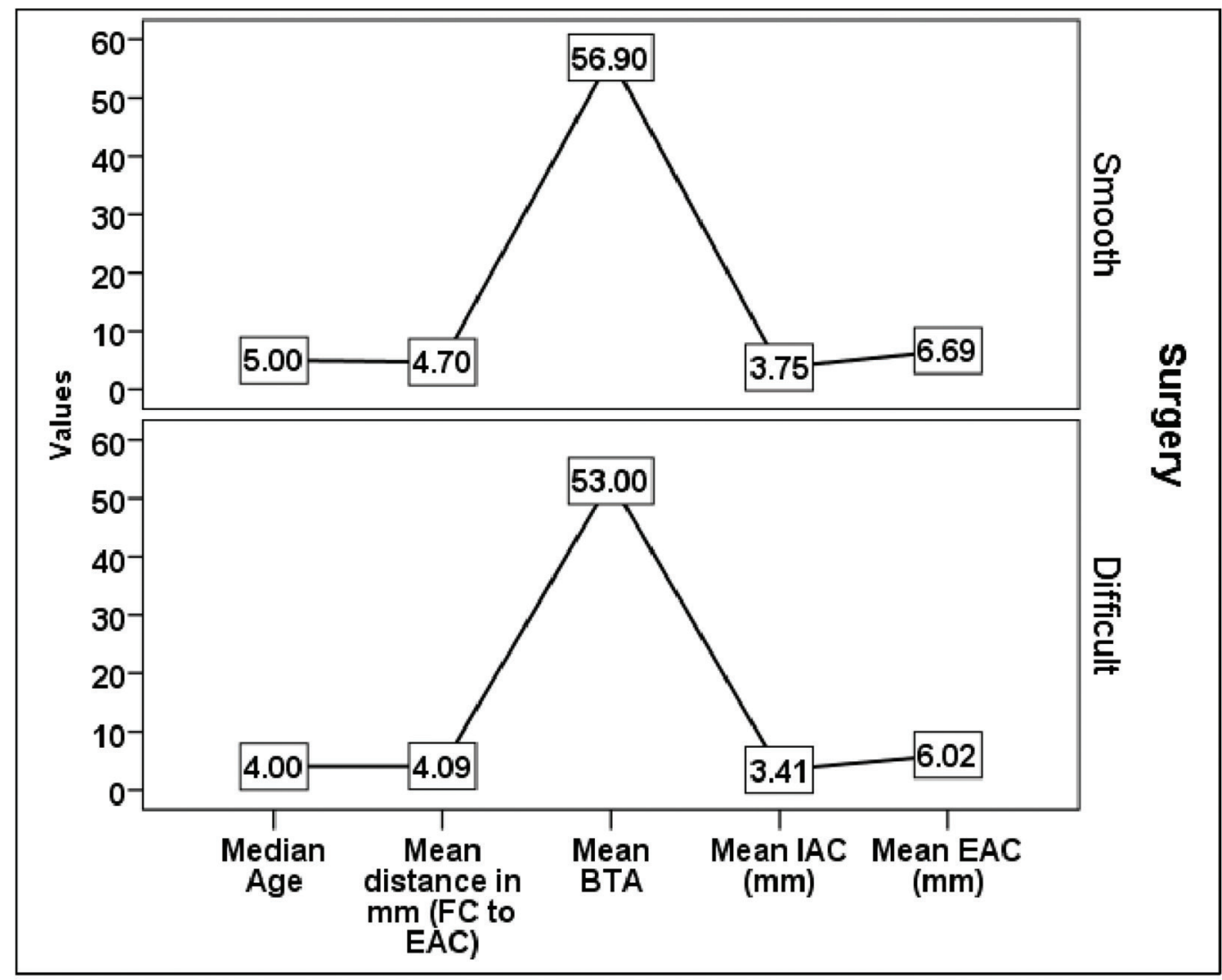

Fig. 7 Line graph showing mean value of age, basal angle, external auditory canal (EAC), distance between the facial canal (FC) and post wall of EAC, and internal auditory canal (IAC) in two surgical feasibility groups. 
of facial canal to EAC ( $p=0.001)$ as well as proportions of poor mastoid pneumatization ( $p=0.002$ ), having high jugular bulb ( $p=0.011)$ and abnormal vestibule/vestibular aqueduct ( $p=0.037$ ), were significantly different between the groups (-Tables 3-5; -Fig. 7).

\section{Association between Surgical Difficulty and Clinical Variables}

In - Table 5, association between the two groups of surgical difficulty and clinical variables was tested using chi-square
test/Fisher's exact test. Result indicated that variables, namely, EAC, BTA, distance between facial canal to EAC, IAC, poor mastoid pneumatization, and abnormal vestibule/ vestibular aqueduct were significantly associated with surgical difficulty $(p<0.05)$.

\section{Predictors of the Surgical Feasibility}

The surgical difficulty and clinical variables, namely, EAC, BTA, distance between facial canal to EAC, IAC, poor mastoid pneumatization, and abnormal vestibule/vestibular

Table 3 Demographic variables and HRCT measurement of clinical variables and its association with surgical outcomes

\begin{tabular}{|l|l|l|l|l|}
\hline Variables & $\begin{array}{l}\text { Total } \\
(N=55)\end{array}$ & $\begin{array}{l}\text { Smooth } \\
(\boldsymbol{n}=\mathbf{4 2})\end{array}$ & $\begin{array}{l}\text { Difficult } \\
(\boldsymbol{n}=\mathbf{1 3})\end{array}$ & $\boldsymbol{p}$-Value \\
\hline BTA & $55.98 \pm 3.43$ & $56.90 \pm 2.26$ & $53 \pm 4.76$ & 0.032 \\
\hline Age & $5(3-14)$ & $5(3-15)$ & $4(2-15)$ & 0.359 \\
\hline Sex (M/F) & $29 / 26$ & $22 / 20$ & $7 / 6$ & 0.926 \\
\hline EAC (mm) & $6.53 \pm 0.77$ & $6.69 \pm 0.69$ & $6.01 \pm 0.80$ & $\mathbf{0 . 0 0 4}$ \\
\hline $\begin{array}{l}\text { Distance between } \\
\text { facial canal and EAC }\end{array}$ & $4.56 \pm 0.55$ & $4.70 \pm 0.51$ & $4.09 \pm 0.39$ & $\mathbf{0 . 0 0 1}$ \\
\hline IAC (mm) & $3.67 \pm 0.54$ & $3.75 \pm 0.57$ & $3.41 \pm 0.33$ & $\mathbf{0 . 0 0 9}$ \\
\hline
\end{tabular}

Abbreviations: BTA, basal turn angle; EAC, external auditory canal; F, female; HRCT, high-resolution computed tomography; IAC, internal auditory canal; $\mathrm{M}$, male.

Note: $\mathrm{p}<0.05$ significant.

Table 4 HRCT and MRI findings and its association with surgical outcomes

\begin{tabular}{|c|c|c|c|c|}
\hline Variables & $\begin{array}{l}\text { Smooth } \\
(n=42)\end{array}$ & $\begin{array}{l}\text { Difficult } \\
(n=13)\end{array}$ & $\begin{array}{l}\text { Total } \\
(N=55)\end{array}$ & $p$-Value \\
\hline Mastoid pneumatization (poor) & $1(2.4)$ & $5(38.5)$ & $6(10.9)$ & 0.002 \\
\hline Middle ear cavity (abnormal) & $2(4.8)$ & $3(23.1)$ & $5(9.1)$ & 0.080 \\
\hline Otosclerosis & $0(0)$ & $2(15.4)$ & $2(3.6)$ & 0.053 \\
\hline High jugular bulb & $0(0)$ & $3(23.1)$ & $3(5.5)$ & 0.011 \\
\hline Sigmoid sinus (abnormal) & $2(4.8)$ & $2(15.4)$ & $4(7.2)$ & 0.234 \\
\hline Vestibule/vestibular aqueduct (abnormal) & $1(2.4)$ & $3(23.1)$ & $4(7.3)$ & 0.037 \\
\hline Cochlea (abnormal) & $1(2.4)$ & $2(15.4)$ & $3(5.5)$ & 0.136 \\
\hline Vestibule & $2(4.8)$ & $2(15.4)$ & $4(7.2)$ & 0.234 \\
\hline Endolymphatic duct/sac & $2(4.8)$ & $3(23.1)$ & $5(9.1)$ & 0.080 \\
\hline
\end{tabular}

Abbreviations: HRCT, high-resolution computed tomography; MRI, magnetic resonance imaging.

Note: Fisher's exact test used, $p<0.05$ significant.

Table 5 Predictors of the difficult surgical process in the cochlear patients $(N=55)$

\begin{tabular}{|l|l|l|l|l|l|l|l|}
\hline \multirow{2}{*}{ Variables } & \multicolumn{3}{|c|}{ Univariate analysis } & \multicolumn{4}{c|}{ Multivariate analysis } \\
\cline { 2 - 9 } & OR & Lower & Upper & AOR & Lower & Upper & $p$-Value \\
\hline EAC & 0.25 & 0.09 & 0.71 & 0.13 & 0.03 & 0.58 & 0.007 \\
\hline BTA & 0.68 & 0.52 & 0.89 & 0.64 & 0.43 & 0.97 & 0.034 \\
\hline Distance between FC and EAC & 0.08 & 0.02 & 0.43 & 0.10 & 0.01 & 0.91 & $\mathbf{0 . 0 4 1}$ \\
\hline IAC (mm) & 0.21 & 0.04 & 0.98 & - & - & - & - \\
\hline Mastoid pneumatization (poor) & 25.63 & 2.63 & 249.72 & - & - & - & - \\
\hline $\begin{array}{l}\text { Vestibule/vestibular } \\
\text { aqueduct (abnormal) }\end{array}$ & 12.30 & 1.15 & 131.10 & - & - & - \\
\hline
\end{tabular}

Abbreviations: AOR, adjusted odds ratio; BTA, basal turn angle; EAC, external auditory canal; FC, facial canal; IAC, internal auditory canal; OR, odds ratio. Note: $p<0.05$ significant. 
aqueduct were significantly associated $(p<0.05)$. Univariate and multivariate binary logistic regression analysis was used to calculate the odds ratio and AOR of the predictors to having surgical difficulty. In univariate binary logistic regression analysis, result showed that higher size of the EAC, BTA, distance between tympanic segment of facial canal to EAC, and IAC were a protecting factor, while poor mastoid pneumatization and abnormal vestibule/vestibular aqueduct were risk factors of the surgical difficulty. In multivariate analysis, out of the six significant factors that were found in univariate analysis, only three variables, namely, higher size of the EAC (AOR $=0.13,95 \% \mathrm{CI}=0.03-0.58, p=0.007)$, higher BTA ( 0.64 , $95 \% \mathrm{CI}=0.43-0.97, p=0.007)$, and higher distance between facial canals to $\operatorname{EAC}(0.10,95 \% \mathrm{CI}=0.01-0.91, p=0.041)$, showed inverse relationship with difficult surgery and were found to be statistically significant ( - Table 5 ).

\section{Diagnostic Accuracy of the Predictors for Surgical Difficulty}

ROC curve was used to test the diagnostic accuracy as well as to find out the appropriate cutoff value of the surgical difficulty predictors. Result showed that distance between tympanic segment of facial canal to EAC (AUC $=88 \%$ ) was the highest predictor of diagnostic accuracy followed by BTA (AUC $=77 \%$ ) and EACs (AUC $=76 \%$ )

( - Fig. 8). For getting balancing sensitivity and specificity of the predictors, cutoff value of 6.25 (sensitivity $=76.9 \%$, specificity $=78.6 \%)$ of the distance between facial canals to EAC, 4.45 (sensitivity $=84.6 \%$, specificity $=81 \%$ ) for BTA, and 55.25 (sensitivity $=61.5 \%$, specificity $=73.8 \%$ ) for EACs were considered as the best.

\section{Discussion}

Cochlear implantation is a standard procedure for the rehabilitation of patients with SNHL. ${ }^{3}$ It has become an accepted treatment in patients with profound SNHL. Radiologists have assumed a larger role in evaluating these patients as the number of procedures increase. ${ }^{4}$ The aim of our study was to evaluate the role of HRCT and MRI in the postoperative assessment of surgical difficulty in cochlear implantation. Various studies have been undertaken to establish the role of CT scan and MRI in preoperative evaluation of cochlear implant candidates and its imaging findings that are likely to affect the eligibility for implantation, risk, and surgical approach. A cross-sectional study was conducted by Lima Júnior et al to investigate the accuracy of imaging studies as predictors of possible complications of surgery. They found that the preoperative radiological evaluation by CT was effective in identifying anatomic abnormalities, allowing surgeons to avoid, or at least be aware of, possible complications. This study demonstrated that CT and MRI were superior to CT alone. ${ }^{5}$ A similar experience was observed in our study, where $\mathrm{CT}$ and MRI were found superior in decision making

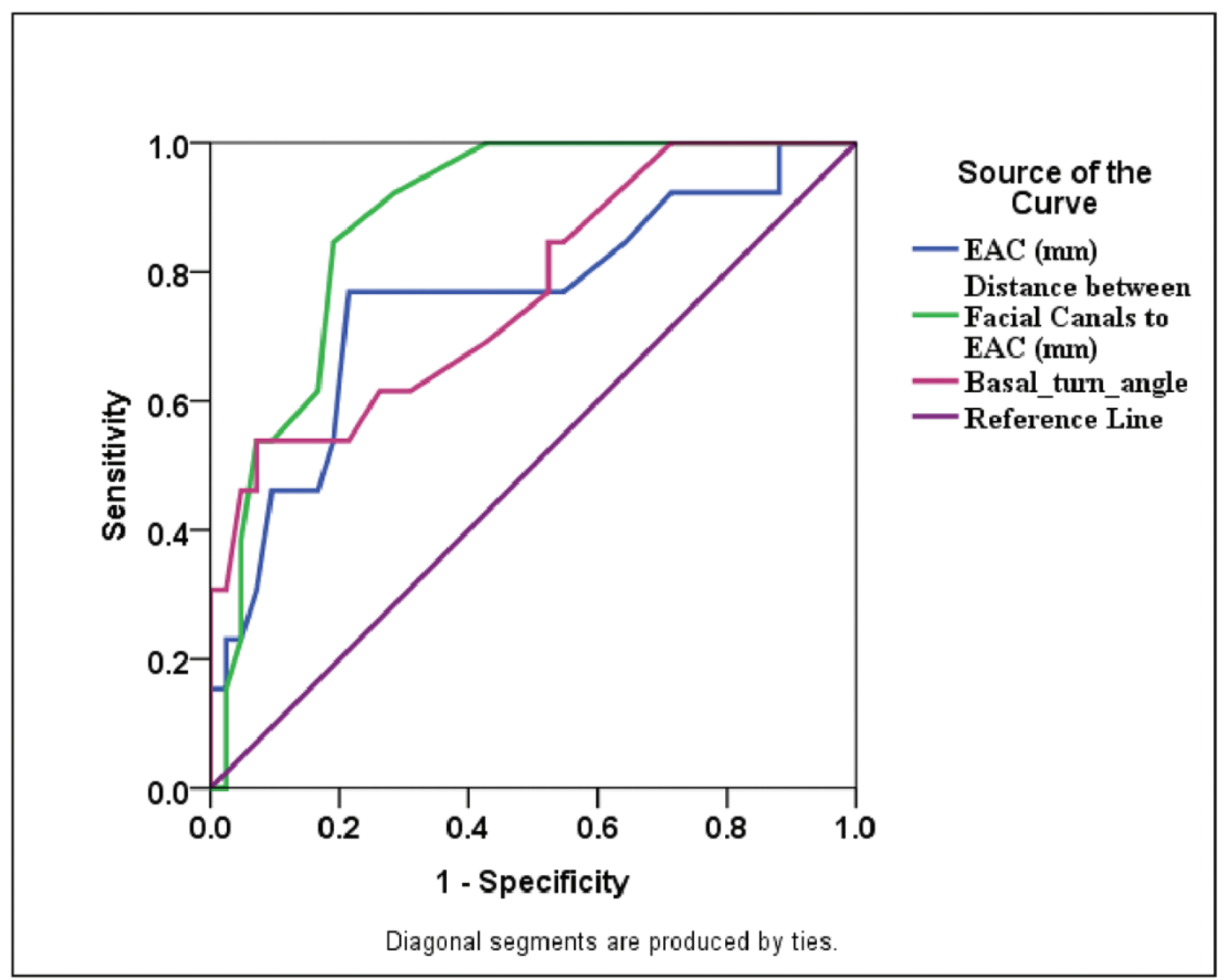

Fig. 8 Area under the curve (of the receiver operating characteristics [ROC] curve) showing comparisons in diagnostic accuracy of the predictors of the surgical difficulty. 
8 Role of High-Resolution Imaging in Cochlear Implant Surgery Tiwari et al.

Table 6 Diagnostic accuracy of the surgical difficulty predictors

\begin{tabular}{|l|l|l|l|l|l|}
\hline Variables & $\begin{array}{l}\text { AUC-ROC } \\
(95 \% \mathrm{Cl})\end{array}$ & $p$-Value & Cutoff & Sensitivity & Specificity \\
\hline EACs in mm & $0.76(0.59-0.92)$ & 0.006 & 6.25 & 76.9 & 78.6 \\
\cline { 4 - 6 } & & & 6.85 & 84.6 & 35.7 \\
\hline $\begin{array}{l}\text { Distance (mm) between } \\
\text { facial canals and EAC }\end{array}$ & $0.88(0.79-0.97)$ & $<0.001$ & 4.55 & 92.3 & 71.4 \\
\hline Basal turn angle & $0.77(0.62-0.92)$ & 0.004 & 57.55 & 84.6 & 81.0 \\
\cline { 3 - 6 } & & & 55.25 & 61.5 & 47.6 \\
\hline
\end{tabular}

Abbreviations: AUC-ROC, area under the curve calculated by receiver operating characteristics curve; $\mathrm{Cl}$, confidence interval; EAC, external auditory canal. Note: Lower values indicate more surgical difficulty, $p<0.05$ significant.

as compared with the CT alone to evaluate the candidacy of the cochlear implants. CT and MRI for evaluation of cochlear implant candidates require consideration of a variety of clinical and radiographic factors. Candidates with SNHL who met established audiologic criteria for cochlear implantation may have unique audiologic, medical, and anatomic characteristics that necessitate special consideration regarding cochlear implantation candidacy and outcome. ${ }^{6,7}$ In our study, $69.1 \%$ patients had no radiological abnormality, while $30.9 \%$ patients showed some type of abnormality including acquired disease and congenital malformation. Note that $18 \%$ $(n=10)$ patients showed various type of congenital inner ear malformation on CT and MRI. Similar result was observed in Chaturvedi et al, where, $16.7 \%$ patients showed various types of congenital inner ear malformations. ${ }^{8}$ In our study, cochlea/ cochlea-vestibular deficiency, endolymphatic duct dilatation, and vestibule/SCC anomaly were found in $12.5,10.5$, and $5.8 \%$, respectively, as compared with 25,12 , and $7 \%$ reported by Lin et al. ${ }^{9}$ Our finding is also comparable with Agarwal et al. ${ }^{10}$ Surgical difficulty was negatively associated with higher size of EAC, distance between tympanic segment of facial canal to EAC, and BTA $(p<0.05)$, which indicated that patients with narrow size of the above parameters increase the difficulty of the surgery while no association was observed with age and sex $(p>0.05)$. Similarly, poor mastoid pneumatization, abnormal middle ear cavity, and abnormal vestibule/vestibular aqueduct were more commonly associated with surgical difficulty $(p<0.05)$. Our finding was comparable with the study conducted by in Wu et al. ${ }^{11}$ Joshi et al also published the role of imaging in preoperative evaluation of cochlear implantation. They found that imaging has important role in the evaluation of congenital SNHL. A broad spectrum of inner ear malformations has been described and linked to development at different stages of embryogenesis, and various systems have been proposed for classifying them. ${ }^{12}$ In our study, ROC curve revealed that distance between facial canals to $\mathrm{EAC}$ (AUC $=88 \%$ ) can be considered as a good predictor of surgical difficulty followed by BTA (AUC $=77 \%$ ) and EACs $($ AUC $=76 \%)$ (-Table 6). Although similar study to predict the surgical difficulty through AUC could not be found.

MRI and CT both have roles in the preoperative assessment of inner ear abnormalities, cochlear nerve deficiency, and variant anatomy that might affect the decision to implant and the prognosis for auditory improvement and increased risk for complications. They might also affect the surgical approach and made implantation difficult. ${ }^{13,14}$ An organized report in cochlear implant patients should provide the surgeon with clear and concise information with special focus on the surgeon's expectations to prepare a clinically relevant report. A constant communication between the imaging specialist and the cochlear implant surgeon improves image interpretation and ensures a successful implantation. ${ }^{15}$

\section{Conclusion}

Anatomy of temporal bone and cochlea by HRCT and MRI are decisive factors in the surgical planning for the cochlear implantation team. Radiologist experienced in the anatomy of temporal bone plays a major role with direct impact on the success of the surgical intervention, helping the surgeon planning the operation and predicting potential complications in cochlear implant candidates. HRCT and MRI are recommended in all patients for preimplant analysis of the temporal bone morphology. BTA and distance between tympanic segment of facial nerve and EAC (midpoint between level of I-S joint corresponding to EAC and exit of chordae tympanic at level of EAC) are important predictors for evaluating intra- and postoperative complications.

\section{Conflict of Interest}

None declared.

\section{References}

1 Maniglia AJ. State of the art on the development of the implantable hearing device for partial hearing loss. Otolaryngol Clin North Am 1996;29(2):225-243

2 Sorkin DL. Sorkin Cochlear implantation in the world's largest medical device market: Utilization and awareness of cochlear implants in the United States. Cochlear Implants Int 2013; 14(Suppl 1):S4-S12. Doi: 10.1179/1467010013Z.00000000076

3 Zaghloul RA, Sheikh ML, Baky FA, Nouem KI. Role of high resolution CT and radiography in postoperative evaluation of cochlear implantation. Egypt J Radiol Nucl Med 2011;42(2):177-184

4 Witte RJ, Lane JI, Driscoll CLW, et al. Pediatric and adult cochlear implantation. Radiographics 2003;23(5):1185-1200

5 Lima Júnior LR, Rocha MD, Walsh PV, Antunes CA, Dias Ferreira Calhau CM. Evaluation by imaging methods of cochlear implant candidates: radiological and surgical correlation. Rev Bras Otorrinolaringol (Engl Ed) 2008;74(3):395-400 
6 Vlastarakos PV, Nikolopoulos TP, Pappas S, Buchanan MA, Bewick J, Kandiloros D. Cochlear implantation update: contemporary preoperative imaging and future prospects - the dual modality approach as a standard of care. Expert Rev Med Devices 2010;7(4):555-567

7 Hang AX, Kim GG, Zdanski CJ. Cochlear implantation in unique pediatric populations. Curr Opin Otolaryngol Head Neck Surg 2012;20(6):507-517

8 Chaturvedi A, Mohan C, Mahajan SB, Kakkar V. Imaging of cochlear implants. Indian J Radiol Imaging 2006;16:385-392

9 Lin $\mathrm{YL}, \mathrm{Wu} \mathrm{CM}$, Toh $\mathrm{CH}$, et al. Magnetic resonance imaging evaluation for predictive factors of cochlear nerve deficiency in pediatric cochlear implant candidates. Radio Sci 2013;38: 43-50

10 Agarwal SK, Singh S, Ghuman SS, Sharma S, Lahiri AK. Radiological assessment of the Indian children with congenital sensorineural hearing loss. Int J Otolaryngol 2014;2014: 808759
11 Wu WJ, He XB, Tan LH, et al. Imaging assessment of profound sensorineural deafness with inner ear anatomical abnormalities. J Otol 2015;10(1):29-38

12 Joshi VM, Navlekar SK, Kishore GR, Reddy K, Kumar ECV. CT and MR imaging of the inner ear and brain in children with congenital sensorineural hearing loss. Radiographics 2012;32: 683-698

13 Tamplen M, Schwalje A, Lustig L, Alemi AS, Miller ME. Utility of preoperative computed tomography and magnetic resonance imaging in adult and pediatric cochlear implant candidates. Laryngoscope 2016;126(6):1440-1445

14 Young JY, Ryan ME, Young NM. Preoperative imaging of sensorineural hearing loss in paediatric candidates for cochlear implantation. Radiographics 2014;34:E133-E149

15 Vaid S, Vaid N. Imaging for cochlear implantation: structuring a clinically relevant report. Clin Radiol 2014;69(7):e9-e24 